\title{
The molecular pathogenesis of Trichilemmal carcinoma
}

\author{
Jeong Hyun Ha ${ }^{1}$, Cheol Lee ${ }^{2}$, Kyu Sang Lee ${ }^{3}$, Chang-sik Pak ${ }^{4}$, Choong-Hyun Sun ${ }^{5}$, Youngil Koh ${ }^{6,7,8^{*+}}$ (DD and \\ Hak Chang ${ }^{9^{*+}}$
}

\begin{abstract}
Background: Trichilemmal carcinoma (TC) is an extremely rare hair follicle tumor. We aimed to explore the genetic abnormalities involved in TC to gain insight into its molecular pathogenesis.

Methods: Data from patients diagnosed with TC within a 12-year period were retrospectively reviewed. Genomic DNA isolated from a formalin-fixed paraffin-embedded (FFPE) tumor tissue block was sequenced and explored for a panel of cancer genes.

Results: DNA was extracted from the FFPE tissue of four patients ( $50 \%$ female; mean age, 51.5 years) diagnosed with TC for analysis. The tumor was located in the head and neck of three patients and in the shoulder of one patient. TP53 mutations (p.Arg213*, p.Arg249Trp, and p.Arg248GIn) were found in three patients. Fusions previously identified in melanoma were detected in two patients (TACC3-FGFR3 and ROS1-GOPC fusions). Other mutations found included NF1-truncating mutation (Arg1362*), NRAS mutation (p.Gln61Lys), TOP1 amplification, and PTEN deletion. Overall, genetic changes found in TC resemble that of other skin cancers, suggesting similar pathogenesis. All patients with TP53 mutations had aggressive clinical course, two who died (OS 93 and 36 months), and one who experienced recurrent relapse.

Conclusions: We reported the genomic variations found in TC, which may give insight into the molecular pathogenesis. Overall, genetic changes found in TC resembled that of other skin cancers, suggesting similar pathogenesis. TP53 mutations was were identified in patients who had an aggressive clinical course. Genetic alterations identified may further suggest the potential treatment options of TC.
\end{abstract}

Keywords: Trichilemmal carcinoma, DNA sequencing, Molecular pathogenesis

\section{Background}

Trichilemmal carcinoma (TC) is a rare hair follicle tumor originating from the outer root sheath epithelium. Although its incidence rate is not clear, it is known to be very rare. According to the review by Hamman et al. [1], only 103 cases has been reported as of 2014. TC was

\footnotetext{
* Correspondence: snuhgo01@gmail.com; prshchang@gmail.com

${ }^{+}$Youngil Koh and Hak Chang contributed equally to this work.

${ }^{6}$ Department of Internal Medicine, Seoul National University Hospital, Seoul, Korea

${ }^{9}$ Department of Plastic and Reconstructive Surgery, Seoul National University College of Medicine, Seoul, Korea

Full list of author information is available at the end of the article
}

first reported by Headington in 1976 [2] and is also known as tricholemmal carcinoma or tricholemmocarcinoma. It is sometimes confused with the malignant form of a proliferating trichilemmal cyst or proliferating trichilemmal carcinoma, but they have distinct features. TC occurs in aged people as a solitary lesion in a sunexposed area, especially the head and neck region. Its clinical features resemble basal cell carcinoma, squamous cell carcinoma, keratoacanthoma, or proliferating pilar cyst $[3,4]$. It manifests as a polypoid or exophytic mass with or without ulceration. It can be also found as an erythematous or keratotic nodule or indurated

(c) The Author(s). 2020 Open Access This article is licensed under a Creative Commons Attribution 4.0 International License, which permits use, sharing, adaptation, distribution and reproduction in any medium or format, as long as you give appropriate credit to the original author(s) and the source, provide a link to the Creative Commons licence, and indicate if changes were made. The images or other third party material in this article are included in the article's Creative Commons licence, unless indicated otherwise in a credit line to the material. If material is not included in the article's Creative Commons licence and your intended use is not permitted by statutory regulation or exceeds the permitted use, you will need to obtain permission directly from the copyright holder. To view a copy of this licence, visit http://creativecommons.org/licenses/by/4.0/ The Creative Commons Public Domain Dedication waiver (http://creativecommons.org/publicdomain/zero/1.0/) applies to the data made available in this article, unless otherwise stated in a credit line to the data. 
plaque. It is thought to be locally aggressive but has a benign clinical course, which can be easily treated with complete excision. Although rare, multiple cases of local recurrence in TC have been reported. In addition, one metastatic tumor has been reported and was treated with adjuvant chemotherapy after wide surgical excision [1]. Typical histopathology of TC shows trichilemmal keratinization and a peripheral palisading pattern indicating the follicular root sheath origin of the tumor. The tumor consists of cytologically atypical, glycogen-rich cells with atypical mitosis. The border is sharply defined and has pushing margins continuous with epidermis or pilosebaceous structures [5].

Its pathogenesis is not well understood, but several factors have been suggested as risk factors including UV and ionizing radiation or preexisting scars. Some reports have described trichilemmal carcinoma diagnosed in patients with xeroderma pigmentosa, those with Cowden syndrome, and solid-organ transplant recipients [6]. Other reports have described TC arising from longstanding seborrheic keratosis [7], wherein histology of the lesion revealed varying degrees of local actinic damage. These reports imply that the development of TC is associated with chronic UV and radiation exposure or aging process. However, other reports have indicated that p53 gene is related with malignant transformation of TC. Takata et al. [8] suggested that the loss of wild-type p53 heterozygosity is a critical event for the malignant transformation of TC. To our knowledge, no other reports have identified the genetic alteration observed in TC.

Genomic profiling is widely performed in various tumors, and the results usually suggest the pathogenesis or treatment options. We aimed to explore the genetic abnormalities involved in TC using panel of cancer genes to obtain insight into its molecular pathogenesis. The results of this study may further suggest possible treatment options or potential drug targets.

\section{Methods}

Clinical data was retrospectively reviewed in six patients who were diagnosed with trichilemmal carcinoma from 2004 to 2016 in Seoul National University Hospital (SNUH) and Seoul National University Bundang Hospital (SNUBH). Of these, four patients underwent a panel sequencing of 83 genes. Other patients were not available for DNA extraction due to the lack of tissue from the formalin-fixed paraffin-embedded (FFPE) tumor tissue block. Overall survival (OS) was calculated as the time from diagnosis of $\mathrm{TC}$ to death from any causes. Patients were censored at the time of the last visit until January 1, 2020. The study was approved by the Institutional Review Board of SNUH and SNUBH (IRB No. H-1703-035-836, B-1711-432-401).

\section{Next-generation sequencing}

DNA was extracted from $10-\mu \mathrm{m}$ thick paraffin sections containing a representative portion of each tumor block by proteinase-K digestion. Genomic DNA was isolated from FFPE tumor tissue blocks using the QIAamp DNA mini kit (Qiagen, Manchester, UK), and the qualified DNA samples were captured and sequenced with SureSelect (Agilent, Inc., USA) following the manufacturer's instructions. The panel of 83 targeted cancer genes were focused on well-known oncogenes reported in the Catalog of Somatic Mutations in Cancer (COSMIC) database rather than relatively unknown genes whose functional effects are currently in question. The panel included the coding exons of 72 genes to detect single nucleotide variants (SNVs), insertion/deletions (indels), and copy number variations (CNVs), as well as some introns for 5 genes to detect gene fusions. After being aligned to the human genome 19 reference and filtered for germline polymorphisms and false positives, SNVs with a variant allele frequency $\geq 5 \%$ and indels with a variant allele frequency $\geq 10 \%$ were selected as the final results. SNVs and indels were detected with the ensemble method integrating three open-source callers: UnifiedGenotyler [9], LoFreq [10], SNVer [11], and SamsungSDS's inhouse callers.

CNVs were analyzed using the depth of coverage for each target region between the tumor and preprocessed normal data. For translocation detection, a paired-end mapping analysis and a split-alignment analysis were applied. All discordant read-pairs with an abnormal insert-size or orientation were screened and softclipping information of the split-reads was investigated as the evidence of the genomic rearrangements. The cut-off values used were $\mathrm{CNs} \geq 7$ for amplification, $\mathrm{CN} 0$ for homo-deletion, and split-read support count $\geq 3$ for translocation. SNVs, indels, CNVs, and translocations were discovered with in-house callers developed by SamsungSDS.

\section{Use of public database as a reference}

We used gene expression data identified from panel sequencing to select possible functional genetic changes. Because TC is a rare disease without easy-to-obtain control samples, we used a public database as a reference. We searched the genetic changes identified in our samples in COSMIC (https://cancer.sanger.ac.uk/cosmic) and cBioPortal (http://www.cbioportal.org/public-portal/ ). All genetic changes identified in our samples were searched in Google Scholar to determine their significance. Genetic alterations were considered significant when referred to more than once in the main text or table of the article, showing the association with cancer development as a somatic mutation. Survival data was 
obtained from the Ministry of the Interior and Safety in South Korea for research purpose.

\section{Results}

Four patients (50\% female; mean age, 51.5 years) diagnosed with TC were identified with available FFPE tumor tissue blocks to extract DNA for analysis. The tumors were located in the head and neck of 3 patients and the shoulder of one patient. The mean overall survival (OS) was 88 months (range, 27-128 months). The mean coverage of all the samples was 286 (range, 32679) (Supplementary Methods). TP53 mutations (p.Arg213*, p.Arg249Trp and p.Arg248Gln) were found in three patients. Fusions previously identified in melanoma were detected in two patients (TACC3-FGFR3 and ROS1-GOPC fusion). Other identified mutations included an NF1-truncating mutation (Arg1362*), an NRAS mutation (p.Gln61Lys), TOP1 amplification, and the PTEN deletion. Among four patients, one experienced relapse; the tumor harbored a SNV in NF1 (Arg1362*) and ROS1-GOPC fusion. The recurred tumor was not available for analysis because the quality of the FFPE tissue block was low lacking DNA. TP53 mutations were identified in three patients. Patients with TP53 mutations had an aggressive clinical course: two patients died (OS 93 and 27 months) and another experienced recurrent relapse. Overall, genetic changes found in $\mathrm{TC}$ resembled that of other skin cancers, suggesting similar pathogenesis (Tables 1, 2, 3, 4).

\section{Case 1 (woman, 36-40): Postauricular TC with NF1 mutation and ROS1 fusion}

A female patient presented with a recurrent mass at the postauricular area after laser therapy a year ago. Histology was consistent with trichilemmal carcinoma and invasion to reticular dermis. Perineural invasion or angiolymphatic invasion was not observed, and resection margins were free from tumor cells. Her TC sample from the recurrent lesion was positive for the NF1 gene mutation (p.Arg1362*) and the ROS1-GOPC fusion gene. However, the FFPE tissue block from the primary tumor was not available for DNA extraction. She was free from recurrence for 6 years but underwent right upper lobe lobectomy for adenocarcinoma of lung (Stage IA1) after 5 years.

\section{Case 2 (male, 41-45): shoulder TC with FGFR3 fusion, PTEN deletion, and NRAS and TP53 mutations}

A male patient visited the clinic with a growing shoulder mass that he first noticed 2 years ago. The mass $(9 \times 5 \times$ $1.7 \mathrm{~cm}$ ) was erythematous and verrucous, accompanied by spontaneous bleeding. The clinical impression was keratoacanthoma or verrucous carcinoma. Histological assessment of the mass indicated dermal invasion. Genetic testing identified the TACC3-FGFR3 fusion gene, PTEN deletion, and the NRAS (p.Gln61Lys) and TP53 (p.Arg213*) mutations. Disease-free survival was 39 months, and the patient expired with an overall survival of 93 months.

\section{Case 3 (female, 46-50): scalp TC with TP53 mutation and TOP1 amplification}

A female patient presented with an exudative nodular lesion of the scalp. She had underlying epidermodysplasia verruciformis $(\mathrm{EV})$, with multiple verruca plana on her whole body including the scalp. In addition, she had a history of chronic hepatitis B and cervical intraepithelial neoplasia. For EV, she had been treated with topical imiquimod, interferon- $2 \alpha$ and acitretin or zinc. The exophytic exudative scalp tumor was diagnosed as trichilemmal carcinoma and showed clear resection margins. She experienced recurrence in multiple regions (scalp and bilateral postauricular area) 33 months after the initial operation. Another recurrent tumor was observed in the scalp and lower leg 7 years after the second operation. She did not undergo adjuvant therapy. The sequencing was performed from the initial specimen, and TP53

Table 1 Patient characteristics

\begin{tabular}{|c|c|c|c|c|c|c|c|c|c|c|c|}
\hline Case & Sex & Age & Location & $\begin{array}{l}\text { Mean target } \\
\text { coverage }\end{array}$ & Tumor depth & $\begin{array}{l}\text { Tumor size } \\
(\mathrm{cm})\end{array}$ & Underlying disease & $\begin{array}{l}\text { OS } \\
\text { (months) }\end{array}$ & $\begin{array}{l}\text { Survival } \\
\text { status }\end{array}$ & $\begin{array}{l}\text { DFS } \\
\text { (months) }\end{array}$ & Relapse \\
\hline 1 & $\mathrm{~F}$ & $36-40$ & Postauricular area & 278.9 & Dermis & $1.4 \times 1.2 \times 0.6$ & & 128 & Alive & 128 & No \\
\hline 2 & $M$ & $41-45$ & Shoulder & 32.2 & Dermis & $9 \times 5 \times 1.7$ & & 93 & Dead & 39 & No \\
\hline 3 & $\mathrm{~F}$ & $46-50$ & Scalp & 151.7 & Subcutis & $1 \times 0.8 \times 0.3$ & $\begin{array}{l}\text { Epidermodysplasia } \\
\text { verruciformis } \\
\text { Chronic hepatitis B } \\
\text { CIN }\end{array}$ & 124 & Alive & 33 & Yes \\
\hline 4 & $M$ & $71-75$ & Temporal area & 679.9 & Bone & $5.2 \times 5.0 \times 3.3$ & $\begin{array}{l}\text { HTN } \\
\text { Osteomyelitis of } \\
\text { thumb } \\
\text { Cataract } \\
\text { BPH }\end{array}$ & 27 & Dead & 15 & Yes \\
\hline
\end{tabular}


Table 2 Genes showing non-synonymous somatic SNVs

\begin{tabular}{|c|c|c|c|c|c|c|c|c|c|}
\hline Case & Chr & Position & Ref & Alt & Transcript & Gene & Effect & AA Change & VAF \\
\hline 1 & 17 & $29,576,111$ & C & $T$ & NM_001042492.2 & NF1 & STOP_GAINED & p.Arg1362* & 2.5 \\
\hline 2 & 1 & $115,256,530$ & G & T & NM_002524.4 & NRAS & $\begin{array}{l}\text { NONSYNONYMOUS } \\
\text { CODING }\end{array}$ & p.Gln61Lys & 36.8 \\
\hline 2 & 17 & $7,578,212$ & G & A & NM_001126112.2 & TP53 & STOP_GAINED & p.Arg213* & 57.1 \\
\hline 3 & 17 & $7,577,536$ & T & A & NM_001126112.2 & TP53 & $\begin{array}{l}\text { NONSYNONYMOUS } \\
\text { CODING }\end{array}$ & p.Arg249Trp & 80.5 \\
\hline 4 & 17 & $7,577,538$ & C & T & NM_001126112.2 & TP53 & $\begin{array}{l}\text { NONSYNONYMOUS } \\
\text { CODING }\end{array}$ & p.Arg248Gln & 13.9 \\
\hline
\end{tabular}

mutation (p.Arg249Trp) and TOP1 gene amplification was identified.

\section{Case 4 (male, 71-75): orbital TC with TP53 mutation}

A male patient showed a mass in the right orbital region. He had underlying hypertension, benign prostate hypertrophy, osteomyelitis of the right thumb, and a history of cataracts surgery in the right eye. The mass was identified in the right frontal and superolateral periorbital area. Grossly, the tumor presented with slight protrusion of the temporal region without ulceration. Magnetic resonance imaging revealed that the lesion had invaded the temporalis muscle with extension into the lateral extraconal orbital cavity, abutting the lateral rectus muscle and involving the right frontal skull and lateral orbital rim. Histological assessment showed trichilemmal keratinization, consistent with trichilemmal carcinoma. Genetic testing of the tumor tissue identified a TP53 mutation (p.Arg248Gln). The patient expired with distant metastasis with overall survival of 27 months.

\section{Discussion}

TC is a rare malignant adnexal neoplasm originating from the outer root sheath, wherein cells demonstrate continuity with epidermis or follicular epithelium [5]. Although the pathogenesis of TC has not been clearly identified, it appears to be related to actinic damage, long-term low-dose irradiation, or malignant transformation of trichilemmoma. Histological assessment of TC reveals varying degrees of local actinic damage. Oyama et al. [7] reported trichilemmal carcinoma from long- standing seborrheic keratosis. In addition, other reports have described TC cases in patients with xeroderma pigmentosum or Cowden syndrome [6], as well as in solid-organ transplant recipients. Some of the genetic alterations identified from our samples are in line with previous reports, enabling us to suggest possible pathogenetic mechanisms of TC.

Takata et al. [8] reported that the total loss of the tumor suppressor gene TP53 may result in malignant transformation in TC. The complete loss of wild-type p53 due to the allelic loss of the short arm of chromosome 17p was suggested as a critical event. TP53 mutations were observed in three samples from our study, supporting the role of TP53 mutations in TC occurrence. PTEN deletion was identified in Case 2, but the patient did not have Cowden syndrome. Cowden syndrome, also called PTEN hamartoma syndrome, is caused by germline PTEN mutation. It develops multiple trichilemmoma, which is known to be a premalignant lesion of TC. O'Hare et al. [6] reported a patient with Cowden disease who developed TC. Acquired PTEN deletion in Case 2 may have played an important role in the development of TC, possibly from trichilemmoma. TOP1 amplification was identified in one patient with underlying EV. EV is characterized by persistent human papillomavirus (HPV) infections, especially the $ß-\mathrm{HPV}$ subtype including HPV-5 and HPV-8. In addition, EV is prone to developing skin cancers [16], most of which contain HPV $[17,18]$. The E6 protein from HPV-5 and HPV-8 decreases the level of ATM serine/threonine protein kinase through p300/ATR signaling, increasing

Table 3 Genes with copy number alterations which are well known to be associated with cancer development and/or progression

\begin{tabular}{lllllll}
\hline Case & Chr & Start & End & Gene & Genetic alteration & \\
\hline 2 & Chr10 & $89,624,156$ & $89,725,266$ & PTEN & Del & \\
& & & & $\begin{array}{l}\text { TC was reported in patients with Cowden syndrome, which is caused by } \\
\text { germline PTEN deletion [6]. }\end{array}$ \\
& & & & $\begin{array}{l}\text { PTEN mutation is found in glioblastoma, endometrial carcinoma, lymphoma, } \\
\text { thyroid, breast, prostate carcinoma, melanoma [12]. }\end{array}$ \\
3 & Chr20 & $39,657,593$ & $39,752,015$ & TOP1 & Amp & $\begin{array}{l}\text { TOP1 amplification was reported in advanced and poor prognostic tumors } \\
\text { in melanoma [13]. }\end{array}$
\end{tabular}


Table 4 Fusion genes with possible functional change (Fusion between gene 1 and gene 2 were observed)

\begin{tabular}{|c|c|c|c|c|c|c|c|c|c|c|}
\hline \multirow[t]{2}{*}{ Case } & \multirow{2}{*}{$\begin{array}{l}\text { Mean } \\
\text { distance }\end{array}$} & \multicolumn{4}{|l|}{ Gene 1} & \multicolumn{4}{|l|}{ Gene 2} & \\
\hline & & Gene & Cytoband & Breakpoint & Transcript & Gene & Cytoband & Breakpoint & Transcript & \\
\hline 1 & 230,509 & ROS1 & $6 q 21-q 22$ & Chr6: 117639431 & NM_002944 & GOPC & $6 q 21$ & Chr6: 117913860 & NM_020399 & $\begin{array}{l}\text { Glioblastoma, } \\
\text { Cholangiocarcinoma, } \\
\text { ovarian cancer, NSCLC [14] }\end{array}$ \\
\hline 2 & 68,411 & TACC3 & 4p16.3 & Chr4: 1736923 & NM_006342 & FGFR3 & 4p16.3 & Chr4: 1806178 & NM_000142 & $\begin{array}{l}\text { Glioblastoma, bladder } \\
\text { urothelial tumors, } \\
\text { nasopharyngeal carcinoma, } \\
\text { head and neck cancer, } \\
\text { cervical cancer [15] }\end{array}$ \\
\hline
\end{tabular}

NSCLC Non-small cell lung cancer

carcinogenic potential after UV exposure [19, 20]. However, TOP1 inhibitor is reported to be effective for ATM mutations. We hypothesize that in this patient, decreased ATM caused by HPV resulted in the carcinogenesis of TC. In such circumstances, TOP1 inhibitor may be used as therapeutic agents. In addition, the association with HPV has been reported in other genetic alterations. FGFR3-TACC3 fusions, found in various solid tumors, were identified in tissues of head and neck squamous cell carcinoma that were also positive for HPV [14]. Moreover, PTEN deletion, identified in Case 2, frequently accompanied HPV-induced squamous cell carcinoma [21]. These findings imply that HPV may play a leading role in TC carcinogenesis.

Our TC cases showed common genetic variants with skin cancers. TP53, NF1, and NRAS are frequently mutated genes identified in malignant melanoma [22]. TP53 mutations in melanoma are correlated with sun exposure $[23,24]$. In addition, TOP1 amplification was reported to be associated with more advanced and poor prognostic tumors in melanoma [12]. Furthermore, TP53 and NRAS mutations have been identified in squamous cell carcinoma. Various skin cancers are reported to develop from seborrheic keratosis; one report reviewed a patient who developed TC in long-standing seborrheic keratosis [7]. Given the shared risk factor, chronic sun exposure, TC may have similar molecular pathogenesis with skin cancers. Although controversial, some reports support the origin of basal cell carcinoma as outer root sheath of hair follicle, similar to TC [25, 26]. This also suggests that carcinogenesis can be induced in outer root sheath cells by UV damage. Despite different cells of origin resulting in different types of cancer, a common carcinogen may cause the same genetic mutation for carcinogenesis.

ROS1-GOPC fusions are well known to be found in glioblastoma, cholangiocarcinoma, ovarian cancer, and non-small cell lung cancer. FGFR3-TACC3 fusions have been reported in glioblastoma, bladder urothelial tumors, nasopharyngeal carcinoma, head and neck cancer, and cervical cancer. Moreover, FGFR3-TACC3 [27] and ROS1-GOPC fusions [28] were reported in melanoma.
For both cases, targeted agents showing therapeutic responses to the respective fusions were introduced. A recent report described a patient with metastatic melanoma harboring GOPC-ROS1 gene fusion who showed clinical response to entrectinib [28]. Therapeutic responses can also be expected in TC cases after the identification of such fusion genes.

Our study nevertheless has several limitations. First, due to its retrospective design, the study was susceptible to selection bias. Of note, we could not obtain detailed follow-up data of case 2: although survival outcome was available from the Ministry of the Interior and Safety in South Korea, cause of death could not be identified. Second, we extracted DNA from FFPE tissue blocks, and fixation and storage process may have caused artifacts and low quality. Third, because we used a gene panel, genes not included in our panel could not be tested, possibly leading to significant implications. Fourth, we did not assess the functional consequences of the identified genetic mutations. Fifth, due to the limited sample size, statistical analysis was not possible. However, due to the rareness of the disease, our findings bear significance. Further studies are warranted to investigate certain molecular pathogenesis of $\mathrm{TC}$ and related therapeutic and prognostic implications.

\section{Conclusions}

We reported the genomic variations found in TC, which may give insight into the molecular pathogenesis. Overall, genetic changes found in $\mathrm{TC}$ resembled that of other skin cancers, suggesting similar pathogenesis. TP53 mutations was were identified in patients who had an aggressive clinical course. Genetic alterations identified may further suggest the potential treatment options of TC.

\section{Abbreviations \\ TC: Trichilemmal carcinoma; FFPE: Formalin-fixed paraffin-embedded: COSMIC: Catalog of Somatic Mutations in Cancer; SNV: Single nucleotide variants; CNV: Copy number variations; OS: Overall survival; \\ EV: Epidermodysplasia verruciformis; HPV: Human papillomavirus}

\section{Acknowledgements}

We thank the Global Science experimental Data hub Center (GSDC) and Korea Research Environment Open NETwork (KREONET) service for data 
computing and network provided by the Korea Institute of Science and Technology Information (KISTI)

\section{Authors' contributions}

J.H.H. contributed in conceptualization, collected clinical data, interpreted the data, wrote the main manuscript text. L.C. contributed in conceptualization data curation, provided resources, reviewing and editing. K.S.L. contributed in conceptualization, provided resources, investigation, reviewing and editing. C.P. provided resources, contributed in investigation, reviewing and editing. C.S. contributed in data curation, performed formal analysis, reviewing and editing. Y.K. contributed in conceptualization, methodology, investigation, supervision, reviewing and editing. H.C. contributed in conceptualization, interpretation of the data, supervision, reviewing and editing. The authors have read and approved the manuscript.

\section{Funding}

This work was supported by the National Research Foundation of Korea (NRF) grant funded by the Korea government (MSIP) (Grant number NRF2018R1A4A1022513). FFPE Sample handling and next generation sequencing was performed using the below funding source.

\section{Availability of data and materials}

The datasets generated and/or analyzed during the current study are available in the SRA repository, [https://www.ncbi.nlm.nih.gov/sra/ PRJNA613537]

\section{Ethics approval and consent to participate}

The study was approved by the Institutional Review Board of Seoul National University Hospital (IRB No. H-1703-035-836, B-1711-432-401) and conducted in accordance with the ethical standards. Waivers of informed consent was approved by the Institutional Review board.

\section{Consent for publication}

Not applicable.

\section{Competing interests}

The authors declare that they have no competing interests.

\section{Author details}

${ }^{1}$ Department of Plastic and Reconstructive Surgery, Seoul Metropolitan Government-Seoul National University Boramae Medical Center, Seoul, Korea. ${ }^{2}$ Department of Pathology, Seoul National University Hospital, Seoul, Korea. ${ }^{3}$ Department of Pathology, Seoul National University Bundang Hospital, Gyeonggi, Korea. ${ }^{4}$ Department of Plastic and Reconstructive Surgery, Asan Medical Center, Seoul, Korea. ${ }^{5}$ GenomeOpinion, Seoul, Korea. ${ }^{6}$ Department of Internal Medicine, Seoul National University Hospital, Seoul, Korea. ${ }^{7}$ Cancer Research Institute, Seoul National University College of Medicine, Seoul, Korea. ${ }^{8}$ Center for Medical Innovation, Seoul National University Hospital, Seoul, Korea. ${ }^{9}$ Department of Plastic and Reconstructive Surgery, Seoul National University College of Medicine, Seoul, Korea.

\section{Received: 30 March 2020 Accepted: 27 May 2020}

Published online: 03 June 2020

\section{References}

1. Hamman MS, Brian Jiang SI. Management of trichilemmal carcinoma: an update and comprehensive review of the literature. Dermatol Surg. 2014;40: 711-7.

2. Headington JT. Tumors of the hair follicle. A review. Am J Pathol. 1976;85: 479-514.

3. Reis JP, Tellechea O, Cunha MF, Baptista AP. Trichilemmal carcinoma: review of 8 cases. J Cutan Pathol. 1993:20:44-9.

4. Boscaino A, Terracciano LM, Donofrio V, Ferrara G, De Rosa G. Tricholemmal carcinoma: a study of seven cases. J Cutan Pathol. 1992;19:94-9.

5. Allee JE, Cotsarelis G, Solky B, Cook JL. Multiply recurrent trichilemmal carcinoma with perineural invasion and cytokeratin 17 positivity. Dermatol Surg. 2003;29:886-9.

6. O'Hare AM, Cooper PH, Parlette HL 3rd. Trichilemmomal carcinoma in a patient with Cowden's disease (multiple hamartoma syndrome). J Am Acad Dermatol. 1997;36:1021-3.
7. Oyama N, Kaneko F. Trichilemmal carcinoma arising in seborrheic keratosis: a case report and published work review. J Dermatol. 2008;35:782-5.

8. Takata M, Rehman I, Rees JL. A trichilemmal carcinoma arising from a proliferating trichilemmal cyst: the loss of the wild-type p53 is a critical event in malignant transformation. Hum Pathol. 1998;29:193-5.

9. Van der Auwera GA, et al. From FastQ data to high confidence variant calls: the genome analysis toolkit best practices pipeline. Curr Protoc Bioinformatics. 2013;43(11 10):11-33.

10. Wilm A, et al. LoFreq: a sequence-quality aware, ultra-sensitive variant caller for uncovering cell-population heterogeneity from high-throughput sequencing datasets. Nucleic Acids Res. 2012;40:11189-201.

11. Wei Z, Wang W, Hu P, Lyon GJ, Hakonarson H. SNVer: a statistical tool for variant calling in analysis of pooled or individual next-generation sequencing data. Nucleic Acids Res. 2011;39:e132.

12. Aguissa-Toure AH, Li G. Genetic alterations of PTEN in human melanoma. Cell Mol Life Sci. 2012;69:1475-91. https://doi.org/10.1007/s00018-011-0878-0.

13. Ryan D, et al. Topoisomerase I amplification in melanoma is associated with more advanced tumours and poor prognosis. Pigment Cell Melanoma Res. 2010;23:542-53. https://doi.org/10.1111/j.1755-148X.2010.00720.x.

14. Davies KD, Doebele RC. Molecular pathways: ROS1 fusion proteins in cancer. Clin Cancer Res. 2013;19:4040-5. https://doi.org/10.1158/10780432.CCR-12-2851.

15. Costa R, et al. FGFR3-TACC3 fusion in solid tumors: mini review. Oncotarget. 2016;7:55924-38. https://doi.org/10.18632/oncotarget.10482.

16. de Oliveira WR, Festa Neto C, Rady PL, Tyring SK. Clinical aspects of epidermodysplasia verruciformis. J Eur Acad Dermatol Venereol. 2003;17:394-8.

17. Clower RV, Hu Y, Melendy T. Papillomavirus E2 protein interacts with and stimulates human topoisomerase I. Virology. 2006;348:13-8.

18. Patel T, Morrison LK, Rady P, Tyring S. Epidermodysplasia verruciformis and susceptibility to HPV. Dis Markers. 2010;29:199-206.

19. Wallace NA, Robinson K, Howie HL, Galloway DA. HPV 5 and 8 E6 abrogate ATR activity resulting in increased persistence of UVB induced DNA damage. PLoS Pathog. 2012;8:e1002807.

20. Prati B, Marangoni B, Boccardo E. Human papillomavirus and genome instability: from productive infection to cancer. Clinics (Sao Paulo). 2018; 73(e539s).

21. Koncar RF, Feldman R, Bahassi EM, Hashemi Sadraei N. Comparative molecular profiling of HPV-induced squamous cell carcinomas. Cancer Med. 2017;6:1673-85.

22. Craig S, Earnshaw CH, Viros A. Ultraviolet light and melanoma. J Pathol. 2018:244:578-85.

23. Stahl PL, et al. Sun-induced nonsynonymous p53 mutations are extensively accumulated and tolerated in normal appearing human skin. J Invest Dermatol. 2011;131:504-8.

24. Ragnarsson-Olding BK, Karsberg S, Platz A, Ringborg UK. Mutations in the TP53 gene in human malignant melanomas derived from sun-exposed skin and unexposed mucosal membranes. Melanoma Res. 2002;12:453-63.

25. Donovan J. Review of the hair follicle origin hypothesis for basal cell carcinoma. Dermatol Surg. 2009;35:1311-23.

26. Youssef KK, et al. Identification of the cell lineage at the origin of basal cell carcinoma. Nat Cell Biol. 2010;12:299-305.

27. Lee J, Lee J, Hong SD, Jang KT, Lee SJ. FGFR3-TACC3: a novel gene fusion in malignant melanoma. Precis Future Med. 2018;2:71-5.

28. Couts KL, et al. Acral lentiginous melanoma harboring a ROS1 gene fusion with clinical response to Entrectinib. Jco Precis Oncol. 2017:1.

\section{Publisher's Note}

Springer Nature remains neutral with regard to jurisdictional claims in published maps and institutional affiliations. 